\title{
Invited Address II
}

\section{ATM Networks: Issues and Challenges Ahead}

\author{
Raj Jain \\ Ohio State University \\ Columbus, Ohio
}

\begin{abstract}
Issues in using ATM cells for very high speed applications will be presented. Ensuring that the users benefit from ATM networks involves several other related disciplines. These will be reviewed. A common misunderstanding about future traffic pattern will be discussed. Issues that may affect the realization of current dream of a seamless world-wide high-speed ATM network will be indicated.
\end{abstract}

